\title{
Equilibrium Dynamics in a Two-Sector Model with Taxes
}

\author{
Salvador Ortigueira * \\ Manuel S. Santos \\ Department of Economics \\ Department of Economics \\ Cornell University \\ Arizona State University \\ Ithaca, NY 14850 USA \\ Tempe, AZ 85287 \\ Email: so37@cornell.edu \\ Email: Manuel.Santos@asu.edu \\ Phone: (607) 255-6338 \\ Phone: (480) 965-0748
}

April 9, 2001

Proposed running head: A model with distortionary taxation.

*Corresponding author. 


\begin{abstract}
In this paper we are concerned with the equilibrium dynamics of a two-sector model of endogenous growth with distortionary taxes. We show that for certain parameters values and tax schemes every equilibrium orbit -except the steady state solution- is non-interior; i.e., there are times in which one of the sectors is inactive. This analysis confirms that in multisector models the set of easily checkable, universal conditions that can guarantee the interiority of equilibrium solutions is rather limited.
\end{abstract}

Keywords: Distortionary taxes; Competitive Equilibrium; Dynamic instability; Noninterior equilibrium orbit.

JEL Classification Numbers: D90; E22. 


\section{Introduction}

In this paper, we are concerned with the equilibrium dynamics of a two-sector model of endogenous growth with taxes. We show that for a certain configuration of parameter values and labor income taxes all equilibrium trajectories -except the balanced growth path- have recurrent periods in which one of the sectors remains inactive. That is, all equilibrium trajectories leave temporarily the region in which both sectors are operating. The setting in which this paradoxical dynamic behavior may occur is quite familiar. Indeed, our model is a simple generalization of the Uzawa-Lucas framework in that both the aggregate good sector and the education sector employ physical and human capital as productive inputs. This version of the model has been extensively considered in the endogenous growth literature, especially to investigate the growth effects of different tax rates (e.g., [6], [12] and [14]). This literature on growth and public finance has mainly focused on the effects of different taxes on the balanced growth equilibrium. The results presented in this paper show that the presence of taxes may give rise to complex equilibrium dynamics, characterized by periods of no production in one of the sectors. Therefore, the stability of the balanced growth path in the economy with taxes becomes a minimal prerequisite in order to draw conclusions from this literature about the long-run effects of taxation.

In the model without tax distortions, the transitional dynamics are now relatively well understood (e.g., [1], [4], [7]). Under constant returns to scale technologies, and a felicity function with a constant elasticity of intertemporal substitution, the model contains at most a unique balanced growth path that is saddle-path stable. Moreover, the dynamical system governing the equilibrium dynamics has a block-recursive structure in which equilibrium prices are determined independently of quantities. Hence, the price of human capital in terms of the physical good can be solved from a single differential equation whose stability properties are dictated by the ranking of factor intensities across

sectors. As an interplay of the Stolper-Samuelson effect, when the aggregate good sector 
is intensive in physical capital and the education sector is intensive in human capital, the relative price of human capital follows a stable process, which indicates that this price will adjust gradually toward its steady-state level. Indeed, a sudden increase in the price of human capital rises wages and lowers physical capital rents. Then, equality of total factor returns entails a subsequent drop in the relative price of human capital. Moreover, as an interplay of the Rybczinski effect, the quantity process is inherently unstable. Indeed, a sudden increase in the quantity of human capital would lead to a rise in production in the education sector and a drop in production in the aggregate good sector. Therefore, convergence to the balanced growth path is only attained for appropriately selected perturbations of prices and quantities. In the opposite case in which the aggregate good sector is human capital intensive and the education sector is physical capital intensive, the price process is unstable and the process governing equilibrium factor quantities is saddle-path stable. Hence, regardless of the factor intensity of each sector, the balanced growth path is always saddle-path stable. A key element to understand the dynamics in the undistorted case is that the factor intensity ranking in terms of factor cost shares, which governs the price behavior, and the ranking in terms of factor quantity ratios, which determines the behavior of production, always coincide.

When asymmetric taxation is introduced, the rankings of factor intensities in terms of cost shares and quantity ratios might not coincide. That is, taxes may affect the equilibrium inputs allocation in such a way that a sector may employ a higher relative quantity of a certain input while the other input commands a higher relative cost share. (A condition for this reversal in the ranking of factor intensities is presented in Bond, Wang and Yip [1] for the case of taxes on capital and labor income, and a subsidy to education.) As a consequence of this factor intensity reversal, the saddle-path stability of the balanced growth path may be lost. Specifically, if for certain tax rates a higher proportion of labor is employed in the educational sector, while physical capital commands a higher income share in that sector, then the balanced growth path is unstable. That is, the factor intensity ranking in terms of cost shares entails that the price process is 
unstable, and the factor intensity ranking in terms of quantity ratios entails that the quantity process is also unstable.

If the model displays a unique, unstable balanced growth path, then it is shown in [13] that there does not exist a Markov equilibrium. Even though the condition for the instability of the balanced growth path might not be empirically plausible in the model under consideration, there are several reasons that justify the analysis of the equilibrium dynamics. First, there are reasons of intellectual curiosity. As such, the results presented in this paper contribute to a better understanding of the dynamics in multisectoral models. Second, since our analysis is conducted under completely standard production functions and preferences, our results can be extended to models in which the non stability of the balanced growth path may arise under more plausible policy parameters.

The non-existence of a Markov equilibrium immediately rises a question about the kind of equilibria displayed by the economy. Since the evolution of the relative price of human capital is solved from a single differential equation which is independent of quantities, and the equilibrium dynamics for quantities is generated by a general two-dimensional, autonomous system of differential equations, there seems to be no scope for the existence of chaotic equilibrium solutions in our framework. Furthermore, as it is shown below that prices and quantities must lie in a compact set, the limiting behavior of equilibria is conformed by periodic and quasi-periodic orbits. As one of our main results, we show that for certain parameter values and tax schemes, every equilibrium orbit (excepting the steady state solution) is non-interior; i.e., there are times in which one of the sectors is inactive. Even though in our model the utility and production functions satisfy the usual Inada-type conditions, the rate of transformation between the two production sectors is always bounded. Hence, this exercise illustrates that in multisector models the set of easily checkable, universal conditions that can guarantee the interiority of equilibrium solutions is rather limited. This result is at odds with common beliefs on the role of Inada conditions, and it should serve then as a warning about the limitations of those conditions in multisectoral models. Our results are especially important for the public 
finance literature. Balanced growth analyses of public policies might be misleading if they are not accompanied by a complete study of the transitional dynamics of the model.

The paper is organized as follows. Section 2 presents the model and discusses two preliminary results which motivate our analysis. Sections 3 contains our main results. Some concluding remarks follow in Section 4. Finally, all proofs are gathered in a separate section.

\section{Notation, the Model and some Preliminaries}

We portray a two-sector model of endogenous growth with physical and human capital accumulation. The available stocks of physical capital, $K$, and human capital, $H$, can be allocated to the production of the physical good, $X$, or to the production of education, $Y$. Production in each sector is determined by a simple Cobb-Douglas, constant returns to scale technology. Thus, let

$$
X=A K_{x}^{\alpha} H_{x}^{1-\alpha}, \quad A>0, \quad 0<\alpha<1
$$

be the quantity produced of the aggregate good using the inputs vector $\left(K_{x}, H_{x}\right)$. Regarding the education sector, let

$$
Y=B K_{y}^{\beta} H_{y}^{1-\beta}, \quad B>0, \quad 0<\beta<1
$$

be the quantity produced of the education good using the inputs vector $\left(K_{y}, H_{y}\right)$.

Let $q$ refer to the price of human capital in terms of the aggregate good. Then, for given physical capital returns, $r$, and wage rates, $w$, profit maximization by firms in each sector implies that

$$
\begin{gathered}
\alpha A K_{x}^{\alpha-1} H_{x}^{1-\alpha} \leq r_{x}, \quad q \beta B K_{y}^{\beta-1} H_{y}^{1-\beta} \leq r_{y} \\
(1-\alpha) A K_{x}^{\alpha} H_{x}^{-\alpha} \leq w_{x}, \quad q(1-\beta) B K_{y}^{\beta} H_{y}^{-\beta} \leq w_{y}
\end{gathered}
$$


Where subscripts refer to the corresponding sector, and equality must hold for each condition whenever the corresponding vector $\left(K_{x}, H_{x}\right)$ or $\left(K_{y}, H_{y}\right)$ is positive.

A representative consumer is also present in this economy. This agent is endowed with $k_{0}$ units of physical capital and $h_{0}$ units of human capital. At each moment in time the agent decides on the outlays to be imputed to consumption, $c$, physical capital investment, $I_{k}$, and human capital investment, $I_{h}$, and on the portfolio allocations of capital goods as specified by the fractions of physical capital, $v$, and of human capital, $u$, to be devoted to the production of the physical good. The remaining fractions of physical and human capital are devoted to the production of the education good. In this economy, there is also a public institution or government that enacts a system of tax rates and subsidies on capital and labor incomes. All physical capital returns are taxed at a single flat-rate, $\tau_{k}$; in contrast, wages are taxed in the aggregate good sector at a rate, $\tau_{h}$, and are subsidized in the education sector at a rate, $\phi_{h}$. The proceeds of taxation are rebated to the representative individual as lump-sum transfers, $T$.

For given paths for good and factor prices and tax rates $\left\{q(t), r_{x}(t), r_{y}(t), w_{x}(t), w_{y}(t)\right.$, $\left.\tau_{k}, \tau_{h}, \phi_{h}\right\}$ the agent chooses $\left\{I_{k}(t), I_{h}(t), c(t), u(t), v(t)\right\}$ so as to solve the following optimization problem

$$
\max \int_{0}^{\infty} \frac{c^{1-\sigma}}{1-\sigma} e^{-\rho t} d t
$$

s.t.

$$
\begin{aligned}
& c+I_{k}+q I_{h} \leq\left(v r_{x}+(1-v) r_{y}\right)\left(1-\tau_{k}\right) k \\
& \quad+\left(u w_{x}\left(1-\tau_{h}\right)+(1-u) w_{y}\left(1+\phi_{h}\right)\right) h+T \\
& \dot{k}=I_{k}-\delta k \\
& \dot{h}=I_{h} \\
& c \geq 0, \quad k \geq 0, \quad h \geq 0, \quad 0 \leq u \leq 1, \quad 0 \leq v \leq 1 \\
& k(0)=k_{0}, h(0)=h_{0} \text { given, } \rho>0, \sigma>0, \delta>0, t \in[0, \infty)
\end{aligned}
$$


Here, $\delta>0$ is the depreciation rate of physical capital; for convenience it is assumed that human capital is not subject to depreciation. Now, the specification of the government budget constraint and the market clearing conditions will complete our characterization of a competitive equilibrium. Revenues from factor taxation in both sectors are simply transferred as additional income to the representative individual

$$
T=\tau_{k} r_{x} K_{x}+\tau_{k} r_{y} K_{y}+\tau_{h} w_{x} H_{x}-\phi_{h} w_{y} H_{y}
$$

Observe that these transfers depend on aggregate variables, and not on the decisions made by the representative agent. Finally, market clearing requires at all times equilibrium in good and factor markets

$$
\begin{array}{cl}
c+I_{k}=X=A K_{x}^{\alpha} H_{x}^{1-\alpha}, & I_{h}=Y=B K_{y}^{\beta} H_{y}^{1-\beta} \\
k=K=K_{x}+K_{y}, & v k=K_{x} \\
h=H=H_{x}+H_{y}, \quad & u h=H_{x}
\end{array}
$$

That is, aggregate quantities chosen by firms must be consistent with the agent's choices.

Formally, a competitive equilibrium for this economy is a path of prices and tax rates $\left\{q(t), r_{x}(t), r_{y}(t), w_{x}(t), w_{y}(t), \tau_{k}, \tau_{h}, \phi_{h}\right\}_{t \geq 0}$, a set of choices for aggregate production $\left\{K_{x}(t), K_{y}(t), H_{x}(t), H_{y}(t), X(t), Y(t)\right\}_{t \geq \mathbf{0}}$, and a set of choices for the representative agent $\left\{I_{k}(t), I_{h}(t), c(t), u(t), v(t)\right\}_{t>0}$ that solve problem $(\mathrm{P})$, and such that conditions $(1)-(7)$ are always satisfied. ${ }^{1}$

A balanced growth path $\{k(t), h(t), c(t), u(t), v(t)\}_{t \geq 0}$ is a competitive equilibrium such that $\{k(t), h(t), c(t)\}_{t \geq 0}$ grow at a constant rate $\nu$, and $\{u(t), v(t)\}_{t \geq 0}$ stay constant. If

\footnotetext{
${ }^{1}$ Observe that this definition can encompass non-interior equilibria where at a certain time either good $X$ or good $Y$ is not produced. For instance, good $Y$ will not be produced if $r_{x} \geq r_{y}$ and $w_{x}\left(1-\tau_{h}\right) \geq$ $w_{y}\left(1+\phi_{h}\right)$ and one of these inequalities is strict.
} 
$\nu>0$, then the balanced growth path is said to be interior. All throughout our analysis, we assume that the balanced growth path is interior. This is a rather mild requirement, which as usual is necessary to study local stability properties from the corresponding linearized equilibrium system.

As is well known, the local stability properties of a balanced growth path bear important information on the equilibrium dynamics of the model. In this paper, we make use of two recent contributions upon which we will develop our results in the next section. First, in the present context, the local stability properties of a balanced growth path have been studied by Bond, Wang and Yip [1]. The following simple reformulation of one of their results (see their Proposition 5) will be useful for our purposes.

Proposition 2.1: Assume that the above economy has an interior balanced growth path. Let $\frac{(1-\beta) \alpha}{\beta(1-\alpha)} \frac{1+\phi_{h}}{1-\tau_{h}}>1>\frac{(1-\beta) \alpha}{\beta(1-\alpha)}$. Then, the balanced growth path is locally unstable.

Note that the present condition for local instability calls for the existence of a relatively higher elasticity of education output with respect to physical capital, i.e. $\beta>\alpha$; and the existence of sufficiently high distortions, either through $\phi_{h}$ or $\tau_{h}$, such that in equilibrium the good sector employs a higher fraction of physical capital. That is, asymmetric taxation on labor income distorts inputs allocations in such a way that reverses the ranking of factor quantity ratios.

In related models with endogenous and productive public spending, the condition for local instability may be independent of tax rates, since only preferences and technology parameters may dictate the stability properties of the balanced growth path (see, e.g., [8] and [11]).

Under the conditions of Proposition 2.1, Santos [13] has shown that there does not exist a Markov equilibrium. In our model, a Markov equilibrium can be defined as a list of functions $\left\{I_{k}, I_{h}, c, u, v\right\}$ that depend on $(k, h)$ such that every trajectory generated by these decision rules is a competitive equilibrium. Since the utility function follows a power law and each production sector features constant returns to scale, there is no restriction of 
generality to impose that functions $I_{k}, I_{h}$, and $c$ be homogeneous of degree one in $(k, h)$, and that functions $u$ and $v$ be homogeneous of degree zero. Then, our state variable is in fact the ratio $m=\frac{k}{h}$, and in a Markov equilibrium the law of motion of variable $m$ can be expressed by a function $\dot{m}=g(m)$. Then, we have the following (see [13]).

Proposition 2.2: Under the conditions of Proposition 2.1, for the economy described in this section there does not exist a Markov equilibrium.

In light of these results, some questions come naturally to the fore: If there does not exist a Markov equilibrium, what can one say about the equilibrium dynamics? What is the evolution of capital stocks, production and prices along an equilibrium trajectory? Observe that if there is a Markov equilibrium specified by a unidimensional rule $\dot{m}=g(m)$, then every equilibrium trajectory will converge to a stationary solution or balanced growth path. And depending on whether or not these stationary solutions are interior, every equilibrium orbit will eventually lie in the interior or may ultimately hit the boundary. Consequently, a necessary condition for our results below on non-interiority of equilibrium solutions [see Propositions 3.2 and 3.6] is the non-existence of a Markov equilibrium. This argument, however, does not extend to discrete-time models or to continuous-time models with multiple endogenous state variables, since in those models the equilibrium dynamics may be rather complex even in the presence of a Markov equilibrium.

\section{Main Results}

As it will become clear below, one of the key features in analyzing the equilibrium dynamics in this model is the allocation of existing inputs, $K$ and $H$, into the two productive sectors, $X$ and $Y$. In the present setting, this allocation problem does not have an interior solution at all times $t \geq 0$, that is, there are times in which one production sector is not active. This non-interiority property -which will be discussed in detail later in this section- precludes the study of the equilibrium dynamics based upon commonly 
used local analysis techniques. Consequently, throughout this section, we are bound to consider the possibility that an equilibrium solution may hit the boundary, and hence our approach has to rely on global aspects of the solution.

Although the global analysis of models with multiple state and control variables may be a difficult task in general, we will prove some preliminary results which render the analysis much more handleable. First of all, Lemma 3.1 shows that there is no any stationary solution other than the interior, unstable balanced growth path. It follows from this simple result (cf., Proposition 3.2) that we can eliminate trajectories along which production of one of the two goods becomes permanently inactive after a given time $t$. Indeed, utility maximization precludes a permanent shut down of the aggregate good production sector. Moreover, when the human capital sector is permanently shut down, then the solution behaves as that of the standard one-sector growth model, and hence there should be convergence to a boundary stationary solution.

Lemma 3.1: The interior balanced growth path is unique, and there is no any other boundary stationary solution.

Proposition 3.2: Every equilibrium path cannot stay at the boundary for all $t \geq 0$.

Once convergence to a boundary solution has been ruled out, we can also eliminate the existence of periodic orbits that never leave the interior. In other words, there cannot exist periodic equilibrium paths along which both sectors are always active.

Lemma 3.3: If there is a periodic equilibrium orbit, then this solution cannot be interior at all times.

The proof of this result relies on the block recursive structure of the dynamical system characterizing the equilibrium solutions [e.g., see the Appendix below]. ${ }^{2}$ Thus, whenever the equilibrium trajectory lies in the interior, the dynamic evolution of the relative price of human capital, $q$, depends only on its own value so that it can be specified by a

\footnotetext{
${ }^{2}$ Bond, Wang and Yip [1] have exploited this recursive property of the solution in their local analysis around the balanced growth path.
} 
differential equation of the form $\dot{q}=f(q)$. This equation has a unique singular point, $0=f\left(q^{*}\right)$. Moreover, for $\beta>\alpha$ this price process is dynamically unstable. Therefore, if an equilibrium trajectory is interior at all times $t \geq 0$, the only plausible solution for the relative price $q$ is to have $q(t)=q^{*}$ for all $t \geq 0$. Then, for interior solutions the dynamics are specified by a simple pair of differential equations [(A.2)-(A.3) below], and as a result it becomes relatively straightforward to rule out the existence of interior periodic orbits.

In our next sequence of preparatory results we establish the existence of upper and lower bounds for the evolution of the ratio of capital stocks, $m$, and for the relative price of human capital, $q$. Consequently, the set of dynamically recurrent points is comprised in a compact region.

Lemma 3.4: Let $m_{\mathbf{0}}>0$. Then, there are bounds $\underline{m}$ and $\bar{m}$ such that for every equilibrium orbit starting at $m_{\mathbf{0}}$ there is $T$ so that $0<\underline{m} \leq m(t) \leq \bar{m}$ for all $t \geq T$.

Lemma 3.5: There are bounds $\underline{q}$ and $\bar{q}$ such that for every equilibrium price path $q(t)$ there is $T$ so that $0<\underline{q} \leq q(t) \leq \bar{q}$ for all $t \geq T$.

We present now our main result, which will be established below for certain configurations of parameter values satisfying the conditions of Proposition 2.1.

Proposition 3.6: There does not exist an interior non-stationary equilibrium solution. That is, excepting the steady-state solution, along any equilibrium trajectory at a certain time one of the sectors must be inactive.

Thus, apart from the steady state solution, no equilibrium trajectory can be generated by system (A.1)-(A.3) below. Therefore, contrary to common wisdom, this result shows that Inada-type conditions are not sufficient to insure the interiority of an equilibrium path in multisector models. Indeed, it should be pointed out that known conditions to guarantee the interiority of equilibrium solutions in multisector models (cf., [3]) appear very restrictive in the present context, as they cannot be linked to explicit parameterizations of our model. Also, note that within our space of parameter values, Proposition 
3.6 will hold for an open set of economies. The inequalities postulated in Proposition 2.1 are the main specific conditions driving the non-existence of a Markov equilibrium and the non-interiority of solutions as stated in Propositions 2.2 and 3.6. And these strict inequalities are preserved for small changes in parameters $\phi_{h}, \tau_{h}, \alpha$ and $\beta$.

We would like to remark that the proof of Proposition 3.6 is specific to the continuoustime framework. By Lemmas 3.4-3.5 every equilibrium path lies in a compact set. Further, as already explained an interior orbit is determined by a two-dimensional system of differential equations. Therefore, the Poincaré-Bendixon theorem implies that all its limit points must be singular points or periodic orbits. An equilibrium path cannot converge to the steady-state solution as this solution is locally unstable (viz. Lemma 3.1). And an interior equilibrium path cannot converge to a periodic orbit as this is ruled out by Lemma 3.3.

By Proposition 3.6, the system of equations governing the law of motion of an equilibrium trajectory will be changing over time (see the Appendix). Since every equilibrium trajectory lies in a compact set, it must converge to a periodic or quasi-periodic orbit. We have not been able to show the existence of limiting exact periodic orbits. It should be observed, however, that by the closing lemma (cf. [9], [10]) if a dynamical system generates a quasi-periodic orbit or $\varepsilon$-cycle, then there must be a nearby dynamical system that generates an exact periodic orbit. Of course, the closing lemma is established under arbitrary $C^{1}$ perturbations of the dynamical system; and it is not clear that these nearby dynamical systems may be generated by changes in the parameter values of our model.

In what follows, we assume that there is an exact periodic orbit. Our purpose now is to explore the dynamic behavior of our economic variables along the cycle. We already know that a periodic orbit cannot always stay in the interior (Proposition 3.6) and that it cannot always stay in the boundary (Proposition 3.2). Then, several questions come to mind concerning when and which sectors are active, and how the relative price of capital and other variables evolve along a periodic orbit. We have been able to prove the following 
results.

Proposition 3.7: Let $q^{*}$ be the value of the relative price of human capital at the balanced growth path. Then, for every periodic orbit there are times $t$ and $t^{\prime}$ such that $q(t)>q^{*}>q\left(t^{\prime}\right)$

Proposition 3.8: Along a periodic orbit there must be a time interval in which the aggregate good sector is the only one active.

Therefore, along a periodic orbit the relative price of human capital must fluctuate around the steady state value $q^{*}$. Also, there are time periods in which the aggregate good sector is the only one in operation. Thus, there are periods in which the economy stops building up the stock of human capital and it is fully devoted to the production of the physical good. A similar dynamic behavior is presented in [5] in a model with inelastic savings. It is unknown to us as to whether there could be time periods in which the human capital sector is the only one in operation. As it becomes clear from the proofs below, however, periods in which the human capital sector is only active lead to more restrictive fluctuations of the relative price $q$ around the steady state value $q^{*}$.

Finally, our last qualitative result is concerned with the comovement of the relative price of human capital, $q$, and the ratio of the capital stocks, $m$, along a periodic orbit when such an orbit is projected on a $(q, m)$ plane.

Proposition 3.9: Along a periodic orbit, variables $q$ and $m$ must move counter clockwise when such an orbit is projected on a $(q, m)$ plane.

The proof of this result follows from the evolution of $q$ and $m$ at two critical points in time, namely, when $q$ reaches its minimum and maximum values. Since at these two dates the equilibrium cannot be interior, it is relatively straigthforward to show that $m$ has to decrease when $q$ attains a maximum value, and it has to increase when $q$ reaches a minimum value. 


\section{Concluding Remarks}

There are three major points that we would like to highlight from this exercise. First, the paper presents some global results about the dynamics of a simple, basic economic model. Most analyses in economics have focused on the dynamics about some stationary solution. We hope that the techniques developed in this paper may foster more complete studies of the dynamic behavior of other multisector economic models.

Second, the paper illustrates that the introduction of simple tax schemes may preclude the existence of a Markov equilibrium, and may lead to pronounced changes in the dynamics of the economic system. Thus, every non-stationary equilibrium orbit cannot always stay in the interior nor can it ultimately stay in the boundary. There are recurrent time periods in which the equilibrium orbit leaves temporarily the region where both sectors are active.

Moreover, these results hold for open subsets of economies, and there does not seem to be a simple, natural way of modifying the structure of the model so as to restore the convergence of equilibrium orbits to stationary solutions. It may very well be that all these effects come along with substantial variations in welfare. Indeed, if the economic system undergoes periodic fluctuations, by the concavity of the utility function it is plausible that the utility level over a cycle is much lower than the one obtained over the balanced growth path. These welfare comparisons may be assessed by numerical simulation. We should note, however, that computation of equilibrium solutions in the present model seems to be a rather challenging endeavor. As a matter of fact, we have not been able to come up with an effective numerical procedure for the computation of equilibrium solutions.

Finally, by way of example the paper confirms that there does not seem to be an easily verifiable, universal set of conditions that would guarantee the interiority of solutions in multisector dynamic economic models. We would like to emphasize that this premise should not be interpreted as that the set of multisector models with interior equi- 
librium solutions is rather "small". As is well understood, there is the important class of economies with a unique globally stable steady state in which all solutions are interior over some sizeable region. Moreover, one could further think of some other models with more complicated dynamics in which over a relevant capture region or ergodic set all equilibrium solutions are interior. Hence, in many situations interiority of solutions may be a fairly mild assumption. Our main claim, however, remains that the interiority assumption seems to be hard to nail down from primitive characteristics of multisector models.

\section{Proofs}

Proof of Lemma 3.1: For simplicity of exposition, we offer here a proof for the case considered in the paper of $\beta>\alpha$. These arguments can be extended to the more general case $\beta \neq \alpha$.

Let $m_{x}=\frac{K v}{h u}$ and $m_{y}=\frac{K(1-v)}{h(1-u)}$. Then, observe that at an interior equilibrium there must be equality of factor returns across sectors. Hence, the following equilibrium conditions must be satisfied.

$$
\begin{aligned}
\left(1-\tau_{k}\right) \alpha A m_{x}^{\alpha-1} & =\left(1-\tau_{k}\right) q \beta B m_{y}^{\beta-1} \\
\left(1-\tau_{h}\right)(1-\alpha) A m_{x}^{\alpha} & =\left(1+\phi_{h}\right) q(1-\beta) B m_{y}^{\beta}
\end{aligned}
$$

It is a consequence of the Stolper-Samuelson effect that as $q$ goes down, then $r_{x}=\alpha A m_{x}^{\alpha-1}$ has to go down.

Moreover, at an interior balanced growth path, we have the intertemporal conditions

$$
\begin{aligned}
\rho+\sigma \nu^{*}+\delta & =\left(1-\tau_{k}\right) \alpha A m_{x}^{* \alpha-1} \\
\rho+\sigma \nu^{*} & =\left(1+\phi_{h}\right)(1-\beta) B m_{y}^{* \beta} \\
& =\frac{\left(1-\tau_{h}\right)(1-\alpha) A m_{x}^{* \alpha}}{q^{*}}
\end{aligned}
$$


And at a boundary steady state, we must have

$$
\begin{aligned}
\rho+\delta & =\left(1-\tau_{k}\right) \alpha A \widehat{m}_{x}^{\alpha-1} \\
\rho & =\frac{\left(1-\tau_{h}\right)(1-\alpha) A \widehat{m}_{x}^{\alpha}}{\widehat{q}}
\end{aligned}
$$

From (10) and (12) we obtain that $r_{x}^{*}>\widehat{r}_{x}$. Hence, $w_{x}^{*}<\widehat{w}_{x}$. It then follows from (11) and (13) that

$$
\hat{q}=\frac{\left(1-\tau_{h}\right) \hat{w}_{x}}{\rho}>\frac{\left(1-\tau_{h}\right) w_{x}^{*}}{\rho+\sigma \nu}=q^{*}
$$

Consequently, $\hat{q}>q^{*}$.

But, we can also establish that $q^{*}>\hat{q}$, which contradicts our previous statement. This claim ensues from the following facts: (a) By the Stolper-Samuelson effect [cf. (8)-(9)] if $r^{*}>\widehat{r}$, then the price $q$ must go down, and furthermore, $(b)$ sector $Y$ is inactive in the boundary steady state.

Remark 5.1: From conditions (8)-(9) it should be observed that if there is activity in both sectors, then the ratios $m_{x}$ and $m_{y}$ are unambiguously determined as functions of $q$. Also, factor returns are unambiguously determined by $q$, since $r_{x}=\alpha A m_{x}^{\alpha-1}$, $r_{y}=q \beta B m_{y}^{\beta-1}, w_{x}=(1-\alpha) A m_{x}^{\alpha}$ and $w_{y}=q(1-\beta) B m_{y}^{\beta}$. We would like to remark that the price determination process is slightly different if only one sector is active. For instance, let us assume that production only occurs in sector $X$. Then, $m_{x}=m$. Consequently, $r_{x}=\alpha A m_{x}^{\alpha-1}$ and $w_{x}=(1-\alpha) A m_{x}^{\alpha}$. Equality of factor returns across sectors implies that $r_{y}=r_{x}$ and $w_{y}=\frac{\left(1-\tau_{h}\right) w_{x}}{\left(1+\phi_{h}\right)}$. Moreover, for fixed $r_{y}$ and $w_{y}$ there is a price $\tilde{q}$ where a profit maximizing firm in the education sector would just break even, and thus conditions (8)-(9) would be satisfied. It follows that sector $Y$ may be inactive for every price $q$ such that $\tilde{q} \geq q>0$. Related conditions are observed when only sector $Y$ is active.

Proof of Proposition 3.2: First, note that the continuity of the control variables precludes the existence of bang-bang solutions in which employment of capital and labor suddenly shifts from one sector to the other [cf., (A.1)-(A.7) below]. There must be a 
transitional period in which both sectors are active.

Also, there cannot be an equilibrium solution in which the good sector remains always inactive. This is ruled out by utility maximization. On the other hand, there cannot be an equilibrium solution in which the education sector remains always inactive, and in which both factors are solely devoted to the production of the good. In this latter case, the equilibrium dynamics would correspond to those of the neoclassical growth model, and so there should be convergence to a boundary steady state. But the existence of such a steady state solution has been ruled out in Lemma 3.1.

Proof of Lemma 3.3: As discussed in the Appendix the equilibrium law of motion of an interior solution is specified by the following equation system:

$$
\begin{aligned}
\frac{\dot{q}}{q} & =\left(1-\tau_{k}\right) \alpha A m_{x}(q)^{\alpha-1}-\left(1+\phi_{h}\right)(1-\beta) B m_{y}(q)^{\beta}-\delta \\
\frac{\dot{e}}{e} & =\left(1-\tau_{k}\right) \alpha A m_{x}(q)^{\alpha-1}-\rho-\delta-B m_{y}(q)^{\beta}\left(1-\frac{m-m_{y}(q)}{m_{x}(q)-m_{y}(q)}\right) \\
\frac{\dot{m}}{m} & =A m_{x}(q)^{\alpha} \frac{m-m_{y}(q)}{m\left(m_{x}(q)-m_{y}(q)\right)}-\frac{e}{m}-\delta-B m_{y}(q)^{\beta}\left(1-\frac{m-m_{y}(q)}{m_{x}(q)-m_{y}(q)}\right)
\end{aligned}
$$

where $e=\frac{c}{h}$. Observe that the right hand-side on (14) only depends on variable $q$, and in fact it is an increasing function of $q$ [cf., conditions (8)-(9) above]. Therefore, the price process is unstable, and $q(t)$ must be equal to $q^{*}$ along an interior solution. Assume now that there is a periodic orbit. We have just concluded that the law of motion of this periodic orbit should be characterized by (15)-(16) evaluated at $q^{*}$.

We now rule out the existence of an interior periodic orbit. Let's write equations (15) and (16) as, $\dot{e}(t)=f(e(t), m(t)), \quad \dot{m}(t)=g(e(t), m(t))$. From the Bendixon theorem, (cf., [2, p. 248]) sufficient conditions for the non existence of a periodic orbit in a given region containing the steady state are that $\Gamma(e(t), m(t))=f_{1}(e(t), m(t))+g_{2}(e(t), m(t)) \neq$ 0 and that this expression does not change sign for all $e(t)$ and $m(t)$ in that region. (Here, subscripts denote partial derivatives.) In our system, $\Gamma(e(t), m(t))$ is equal to 
$a_{1}+a_{3}+3 a_{2} m(t)$, where constants $a_{1}, a_{2}$ and $a_{3}$ are given by

$$
\begin{aligned}
a_{1} & =\left(1-\tau_{k}\right) \alpha A m_{x}\left(q^{*}\right)^{\alpha-1}-\rho-\delta-B m_{y}\left(q^{*}\right)^{\beta}-\frac{B m_{y}\left(q^{*}\right)^{1+\beta}}{m_{x}\left(q^{*}\right)-m_{y}\left(q^{*}\right)} \\
a_{2} & =\frac{B m_{y}\left(q^{*}\right)^{\beta}}{m_{x}\left(q^{*}\right)-m_{y}\left(q^{*}\right)} \\
a_{3} & =\frac{A m_{x}\left(q^{*}\right)^{\alpha}}{m_{x}\left(q^{*}\right)-m_{y}\left(q^{*}\right)}-\delta-B m_{y}\left(q^{*}\right)^{\beta}-\frac{B m_{y}\left(q^{*}\right)^{1+\beta}}{m_{x}\left(q^{*}\right)-m_{y}\left(q^{*}\right)}
\end{aligned}
$$

Since $\Gamma$ is independent of $e(t)$ and incresing in $m(t)$, it is sufficient to show that this expression is positive when it is evaluated at the minimum $m(t)$ that could be attained if a periodic orbit would exist. When this minimum value of $m(t)$, say $m_{\min }$, is reached, we have $\dot{m}(t)=0$, and therefore it lies on the isocline $\dot{m}(t)=0$, as defined by (16). Since for non-negative values of $e(t)$ and $m(t)$ the right-hand side of $(16)$ is decreasing in $e(t)$ and increasing in $m(t)$, we can safely pick as $m_{\text {min }}$ the corresponding value given by $(16)$ for $\dot{m}(t)=0$ and $e(t)=0$. This latter value is,

$$
m_{\text {min }}=-\frac{a_{3}}{2 a_{2}}+\frac{\left(a_{3}^{2}+4 a_{2} a_{4}\right)^{1 / 2}}{2 a_{2}}
$$

where

$$
a_{4}=\frac{A m_{x}\left(q^{*}\right)^{\alpha} m_{y}\left(q^{*}\right)}{m_{x}\left(q^{*}\right)-m_{y}\left(q^{*}\right)}
$$

Thus, a sufficient condition for the non existence of periodic orbits is,

$$
\Gamma\left(m_{\min }\right)=a_{1}+a_{3}+3 a_{2} m_{\min }>0
$$

Since our purpose is to show that the lemma holds true for a certain configuration of parameter values, we just consider the following parameterization: $A=1, B=0.1, \alpha=$ $0.3, \beta=0.35, \rho=0.05, \delta=0.1, \tau_{k}=0, \tau_{h}=0.2$.

We now compute $\Gamma\left(m_{m i n}\right)$ for different values of $\phi_{h}$ satisfying the condition in Proposition 2.1 . 


\begin{tabular}{cl}
$\phi_{h}$ & $\Gamma\left(m_{\text {min }}\right)$ \\
\hline 0.2 & 4.3389 \\
\hline 0.3 & 3.0602 \\
\hline 0.4 & 2.4367 \\
\hline 0.5 & 2.0712 \\
\hline
\end{tabular}

As a matter of fact, for all computations that we have examined, $\Gamma\left(m_{\min }\right)$ has proved to be positive for all feasible values of $\phi_{h}$.

Proof of Lemma 3.4: It follows from the physical laws of motion [see (16), and (A.9), (A.12) below] that there is an upper bound $\bar{m}$ such that for every feasible solution $m(t)$ with $m(t) \geq \bar{m}$ we must have $\dot{m}(t)<0$. Therefore, $m(t) \leq \bar{m}$ for all sufficiently large $t$.

Regarding the existence of $\underline{m}>0$, observe that depending on which sector is active, prices obey the following relations:

$$
\begin{array}{cl}
\dot{\mu}=\mu(\rho+\delta)-\mu f^{\prime}\left(m_{x}\right), & \dot{\mu}=\mu(\rho+\delta)-\lambda \varphi^{\prime}\left(m_{y}\right) \\
\dot{\lambda}=\lambda(\rho)-\mu f_{w}^{\prime}\left(m_{x}\right), & \dot{\lambda}=\lambda(\rho)-\lambda \varphi_{w}^{\prime}\left(m_{y}\right)
\end{array}
$$

Here, $\mu$ is the shadow price of the physical good, $\lambda$ is the shadow price of the educational good, so that $q=\frac{\lambda}{\mu}$ is the relative price of human capital; also, $f^{\prime}\left(m_{x}\right)$ denotes $(1-$ $\left.\tau_{k}\right) \alpha A m_{x}^{\alpha-1} ; f_{w}^{\prime}\left(m_{x}\right)$ denotes $\left(1-\tau_{h}\right)(1-\alpha) A m_{x}^{\alpha} ; \varphi^{\prime}\left(m_{y}\right)$ denotes $\left(1-\tau_{k}\right) \beta B m_{y}^{\beta-1}$, and $\varphi_{w}^{\prime}\left(m_{y}\right)$ denotes $\left(1+\phi_{h}\right)(1-\beta) B m_{y}^{\beta}$.

Thus, if the lemma is not true, then $m=0$ must be a cluster point for some equilibrium orbit $\{m(t)\}_{t=0}^{\infty}$ with $m(0)=m_{\mathbf{0}}$, where without loss of generality $m_{\mathbf{0}}$ is assumed to be arbitrarily small. Hence, there is an equilibrium orbit with $m(0)=m_{0}$ that will approach zero and then will take an arbitrary amount of time $t$ so that $m(t) \geq m_{0}$. This is because either $m(t)$ is always less than $m_{0}$ or else from the physical law of motion [see (16) and (A.9)] we get that $\dot{m}$ is close to zero for $m$ small enough.

Note that $\frac{\dot{c}}{c}=-\frac{\dot{u}}{\mu}$. Then, one can see from (17)-(18) that during this long period of time, consumption is growing at an arbitrarily large rate in all three possible cases 
(a) When both sectors are active, since both laws of motion in (17) apply, and $\frac{\dot{\mu}}{\mu}$ is therefore arbitrarily high.

(b) When the human capital sector is active, since $\frac{\lambda}{\mu}$ must be arbitrarily high, and $\dot{\mu}=$ $\mu(\rho+\delta)-\lambda \varphi^{\prime}(m)$.

(c) When the aggregate good sector is the only one active, since $\dot{\mu}=\mu(\rho+\delta)-\mu f^{\prime}(m)$.

From these dynamic laws of motion, it follows that initial consumption, $c_{0}$, must be arbitrarily close to zero. However, from the agent's budget constraint, we now show that consumption has a positive lower bound.

Assuming that the agent has one unit of human capital, budget-constrained utility maximization implies that

$$
\frac{c_{0}}{\rho} \geq q+m_{0}
$$

where $m_{\mathbf{0}}$ is the initial amount of physical capital, and it is implicitly assumed that the subsidy to educational labor is sufficiently small so that $T_{t} \geq 0$ for all $t$. Observe that $\frac{\dot{c}}{c}=r_{t}-\rho$ for all $t$. Then, it follows that $c_{t}=c_{0} \exp (R(t)-\rho t)$, where $R(t)=\int_{0}^{t} r(s) d s$. Hence, $c_{t} \exp (-R(t))=c_{0} \exp (-\rho t)$. Therefore, the sum of consumption is precisely $\frac{c_{0}}{\rho}$.

Proof of Lemma 3.5: The bounds $\bar{m}$ and $\underline{m}$ established from the previous proposition will also bound the interest and wages rates. Hence, $q(t)$ must be bounded, as the price of human capital is the discounted sum of wages.

Proof of Proposition 3.6: Since $\dot{q}$ is increasing in $q$ [cf. equation (14)], the price $q$ must be constant along an interior solution. Also, $m \geq 0, e \geq 0$. Then, it follows from the preceding results that both $m$ and $e$ must be contained in a compact set. Hence, by the Poincaré-Bendixon theorem (cf. [2, p. 248]) an interior solution must converge to either a stationary solution or to a closed orbit. But from a careful look at the proof of Lemma 3.3 one can see that dynamical system (14)-(16) cannot contain a periodic orbit for $q$ constant and $0 \leq u \leq 1$. Moreover, an equilibrium trajectory cannot converge to a 
steady state since the unique stationary solution is locally unstable. Therefore, we can conclude that for every equilibrium trajectory there is a time $t \geq 0$ in which one of the production sectors will be inactive.

The proof of Proposition 3.7 is a simple consequence of the following two lemmas:

Lemma 5.2: If there is a periodic orbit, we cannot have $q(t) \geq q^{*}$ for all $t$.

Proof: Let us consider the following cases:

(a) At dates in which the solution is interior, it follows from (14) that $\dot{q} \geq 0$.

(b) If the aggregate good sector is the only one active, then it follows from (17) - (18) that

$$
\frac{\dot{q}}{q}=\frac{\dot{\lambda}}{\lambda}-\frac{\dot{\mu}}{\mu}=-\frac{1}{q} f_{w}^{\prime}(m)+f^{\prime}(m)-\delta
$$

Observe that in this case $q$ must be less than or equal to that price $\tilde{q}$ that would correspond if both sectors are active (cf. Remark 5.1). Therefore, $\tilde{q} \geq q \geq q^{*}$. We now claim that $m<$ $m_{x}\left(q^{*}\right)$; for if $m=m_{x}\left(q^{*}\right)$ for a certain time interval, then consumption $c$ would grow at the steady-state rate and physical capital would be constant, and this is not possible. Therefore, $m<m_{x}\left(q^{*}\right)$ for basically all dates in which the aggregate good sector is the only one active. Then, by virtue of (19), it must be the case that $\dot{q}>0 .^{3}$

(c) If the educational sector is the one active, then it follows from (17)-(18) that

$$
\frac{\dot{q}}{q}=\frac{\dot{\lambda}}{\lambda}-\frac{\dot{\mu}}{\mu}=-\varphi_{w}^{\prime}(m)+q \varphi^{\prime}(m)-\delta
$$

If the human capital sector is the only one active, by continuity of solutions it must be that both sectors were originally active. At the time of switching all production to the human capital sector, we must have $m \leq m_{y}\left(q^{*}\right)$. Also, from then on, $\dot{m}<0 .{ }^{4}$ Thus, it follows from $(20)$ that $\dot{q}>0$.

\footnotetext{
${ }^{3}$ Another way to see this is to write from (19), the equation $\dot{q}=-f_{w}^{\prime}(m)+q\left[f^{\prime}(m)-\delta\right]$, and note that $f^{\prime}\left(m_{x}\left(q^{*}\right)\right)-\delta>0, q>q^{*}$ and $m_{x}\left(q^{*}\right)>m$.

${ }^{4}$ Observe that if the human capital sector is the only one active, we must have $\frac{\dot{m}}{m} \leq-\frac{\dot{h}}{h}<0$
} 
Therefore, in all cases we obtain $\dot{q} \geq 0$ with strict inequality in $(b)-(c)$. But by Lemma 3.3 this is not possible along a periodic orbit.

Lemma 5.3: If there is a periodic orbit, we cannot have $q(t) \leq q^{*}$ for all $t$.

Proof: The strategy of the proof is slightly different to that of the preceding lemma. (a) At dates in which the solution is interior, it follows from (14) that $\dot{q} \leq 0$.

(b) If the human capital sector is the only one active, then $q$ must obey (20). For $\tilde{q}$ as defined above, we must then have $\tilde{q} \leq q$. Since $\dot{m}<0$ and $q \leq q^{*}$, and eventually both sectors must be active, it follows from $(20)$ that $\dot{q}<0$ for basically all $t$.

(c) If the aggregate good sector is the only one active, then $q$ must obey (19). Originally, both sectors were active (cf., Proposition 3.2), and at the time in which all production switches to the aggregate good sector we must have $m \geq m_{x}\left(q^{*}\right)$, and so $\dot{q} \leq 0$. But in view of the above cases $(a)$ and $(b)$, we need to have a date in which $\dot{q}\left(t^{\prime}\right) \geq 0$. By $(19)$, at such a first date we need to have $m_{x}\left(q^{*}\right) \geq m_{x}\left(t^{\prime}\right)=m\left(t^{\prime}\right)$, and it must be the case that $\dot{m}=\dot{K} \leq 0$; also (17) implies that $\frac{\dot{c}}{c} \geq \nu^{*}$. Therefore, from $t^{\prime}$ on we have that $\dot{c} \geq 0$, and so $\dot{m}<0$, until we switch to production in both sectors. But this would be impossible, as $m_{x}\left(q^{*}\right)>m$ and by (8)-(9) production in both sectors at the date of switching requires $q>q^{*}$.

Proof of Proposition 3.8: Assume that there is not such a time interval, and whenever there is only one active sector this is the sector devoted to education. Then, it follows from part $(c)$ of Lemma 5.2, that along some interior interval (when both sectors are active) we must have $q<q^{*}$. (In the opposite case, if $q>q^{*}$, then when we switch to production in the human capital sector we have that $\dot{q}>0$. This means that $\dot{q}>0$ all the time, and this cannot be true along a periodic orbit.) Therefore, we must have $q<q^{*}$ over interior intervals. But notice that from the previous proposition there should be a non interior interval $\left[t_{\mathbf{0}}, t_{1}\right]$ with the property that $q\left(t_{\mathbf{0}}\right)<q^{*}, q\left(t_{1}\right)<q^{*}$ and $q(t)>q^{*}$ for some $t$ in $\left(t_{0}, t_{1}\right)$. The law of motion of $q$ is described by (20). But once $\dot{q}>0$, it follows 
from this equation that $\dot{q}>0$ all the time, as $\dot{m}<0$ in this time interval in which the human capital sector is the only one which is active.

Proof of Proposition 3.9: Let's denote by $q_{\min }$ and $q_{\max }$ the minimum and the maximum values for $q$ along a periodic orbit, and by $t_{0}$ and $t_{1}$ the times where these values are attained. Therefore, $\dot{q}\left(t_{0}\right)=0$ and $\dot{q}\left(t_{1}\right)=0$; moreover, since $q_{\min }$ is the minimum value for $q$, it follows that $\ddot{q}\left(t_{0}\right) \geq 0$. By a similar argument we must have that $\ddot{q}\left(t_{1}\right) \leq 0$. It is readily shown [see equation (14)] that the equilibrium cannot be interior at $t_{0}$ and $t_{1}$.

In order to prove that the orbit must move counter clockwise, we assume that it moves clockwise and find a contradiction. Under this last assumption, we must have $\dot{m}\left(t_{0}\right)>0$ and $\dot{m}\left(t_{1}\right)<0$. If the output sector is the only one active, then from equation (A.8) in the Appendix we obtain that,

$$
\frac{\ddot{q}\left(t_{i}\right)}{q\left(t_{i}\right)}=\frac{\dot{m}\left(t_{i}\right)}{m\left(t_{i}\right)}\left[-\alpha(1-\alpha)\left(1-\tau_{h}\right) A \frac{m\left(t_{i}\right)^{\alpha}}{q\left(t_{i}\right)}-(1-\alpha)\left((1-\alpha)\left(1-\tau_{h}\right) A \frac{m\left(t_{i}\right)^{\alpha}}{q\left(t_{i}\right)}+\delta\right)\right]
$$

for $i=0,1$. Since the expression within square brackets is always negative, the sign for $\frac{\ddot{q}\left(t_{i}\right)}{q\left(t_{i}\right)}$ given by this equation is in contradiction with the sign obtained above, both at $t_{\mathbf{0}}$ and $t_{1}$.

If the human capital sector is the only active, then from equation (A.11) in the Appendix we obtain that,

$$
\frac{\ddot{q}\left(t_{i}\right)}{q\left(t_{i}\right)}=\frac{\dot{m}\left(t_{i}\right)}{m\left(t_{i}\right)}\left[-\beta(1-\beta)\left(1+\phi_{h}\right) B m\left(t_{i}\right)^{\beta}-\beta(1-\beta)\left(1-\tau_{k}\right) B m\left(t_{i}\right)^{\beta-1} q\left(t_{i}\right)\right]
$$

Again, the expression inside the square brackets is negative, and therefore the sign for $\frac{\ddot{q}\left(t_{i}\right)}{q\left(t_{i}\right)}$ is in contradiction with the one obtained above, both at $t_{0}$ and $t_{1}$.

Since these arguments apply to all times in which there is a local minimum or a local maximum for $q$, our proof rules out the existence of trajectories with some clockwise part (including loops), in a $(q, m)$ plane. That is, there cannot exist any time $t$ in which $\dot{q}(t)=0, \ddot{q}(t) \leq 0$ and $\dot{m}(t)<0$, or $\dot{q}(t)=0, \ddot{q}(t) \geq 0$ and $\dot{m}(t)>0$. 
From the above arguments we may conclude that the orbit must move counter clockwise. 


\section{Appendix}

This appendix collects some equilibrium conditions frequently invoked in our analysis. Formal discussions of these conditions can be found in [1], [8] and [11].

Let $q$ refer to the relative price of the education good. Let $m=\frac{K}{h}$, and $e=\frac{c}{h}$. Also, let $m_{x}=\frac{K v}{h u}$, and $m_{y}=\frac{K(1-v)}{h(1-u)}$.

The following conditions must be satisfied along an interior equilibrium:

$$
\begin{aligned}
u(t) & =\frac{m(t)-m_{y}(t)}{m_{x}(t)-m_{y}(t)} \\
v(t) & =\frac{\alpha(1-\beta)\left(1+\phi_{h}\right) u(t)}{\beta(1-\alpha)\left(1-\tau_{h}\right)(1-u(t))+\alpha(1-\beta)\left(1+\phi_{h}\right) u(t)} \\
\frac{\dot{q}(t)}{q(t)} & =\alpha\left(1-\tau_{k}\right) A m_{x}(t)^{\alpha-1}-(1-\beta)\left(1+\phi_{h}\right) B m_{y}(t)^{\beta}-\delta \\
\frac{\dot{m}(t)}{m(t)} & =A m_{x}(t)^{\alpha} \frac{u(t)}{m(t)}-\frac{e(t)}{m(t)}-\delta-B m_{y}(t)^{\beta}(1-u(t)) \\
\frac{\dot{e}(t)}{e(t)} & =\alpha\left(1-\tau_{k}\right) A m_{x}(t)^{\alpha-1}-\rho-\delta-B m_{y}(t)^{\beta}(1-u(t))
\end{aligned}
$$

where $m_{x}(t)$ and $m_{y}(t)$ are solved from the following equations system,

$$
\begin{gathered}
\left(1-\tau_{k}\right) \alpha A m_{x}(t)^{\alpha-1}=\left(1-\tau_{k}\right) q(t) \beta B m_{y}(t)^{\beta-1} \\
\left(1-\tau_{h}\right)(1-\alpha) A m_{x}(t)^{\alpha}=\left(1+\phi_{h}\right) q(t)(1-\beta) B m_{y}(t)^{\beta}
\end{gathered}
$$

If the human capital sector remains inactive the following equilibrium conditions must hold:

$$
\frac{\dot{q}(t)}{q(t)}=-(1-\alpha)\left(1-\tau_{h}\right) A \frac{m(t)^{\alpha}}{q(t)}-\delta+\alpha\left(1-\tau_{k}\right) A m(t)^{\alpha-1}
$$




$$
\begin{aligned}
& \frac{\dot{m}(t)}{m(t)}=A m(t)^{\alpha-1}-\frac{e(t)}{m(t)}-\delta \\
& \frac{\dot{e}(t)}{e(t)}=\alpha\left(1-\tau_{k}\right) A m(t)^{\alpha-1}-\rho-\delta
\end{aligned}
$$

If the final output sector remains inactive the following equilibrium conditions must hold:

$$
\begin{aligned}
& \frac{\dot{q}(t)}{q(t)}=-(1-\beta)\left(1+\phi_{h}\right) B m(t)^{\beta}-\delta+\beta\left(1-\tau_{k}\right) B m(t)^{\beta-1} q(t) \\
& \frac{\dot{m}(t)}{m(t)}=-\frac{e(t)}{m(t)}-\delta-B m(t)^{\beta} \\
& \frac{\dot{e}(t)}{e(t)}=\beta\left(1-\tau_{k}\right) B m(t)^{\beta-1} q(t)-\rho-\delta-B m(t)^{\beta}
\end{aligned}
$$




\section{References}

[1] E. Bond, P. Wang and C. Yip, A general two-sector model of endogenous growth with human and physical capital: Balanced growth and transitional dynamics, $J$. Econ. Theory 68 (1996), 149-173.

[2] M. Hirsch and S. Smale, "Differential equations, dynamical systems, and linear algebra," Academic Press, New York, 1974.

[3] T. Kehoe, D. Levine and D. Romer, Steady states and multiplicity of equilibria in economies with infinitely lived agents, in "Joan Robinson and Modern Economic Theory" (G. Feiwel, Ed.), 521-44, Macmillan Press, London, 1988.

[4] A. Ladrón-de-Guevara, S. Ortigueira and M. Santos, Equilibrium dynamics in twosector models of endogenous growth, J. Econ. Dynam. Control 21 (1997), 115-143.

[5] K. Matsuyama, Growing through cycles, Econometrica 67 (1999), 335-347.

[6] E. McGrattan and J. Schmitz, Explaining cross-country income differences, in "Handbook of Macroeconomics" (J. Taylor and M. Woodford, Eds.), North-Holland, Amsterdam, 1999.

[7] C. Mulligan and X. Sala-i-Martin, Transitional dynamics in two-sector models of endogenous growth, Quart. J. Econ. 108 (1993), 739-775.

[8] S. Ortigueira, Equilibrium indeterminacy in an endogenous growth model: Debt as a coordination device, manuscript, Cornell University, (1999).

[9] C. Pugh, The closing lemma, Am. J. Mathematics 89 (1967a), 956-1009.

[10] C. Pugh, An improved closing lemma and a general density theorem, Am. J. Mathematics 89 (1967b), 1010-1021. 
[11] X. Raurich, Indeterminacy and government spending in a two-sector model of endogenous growth, Rev. Econ. Dynam. 4 (2000), 210-230.

[12] S. Rebelo, Long-run policy analysis and long-run growth, J. Polit. Economy 99 (1991), 500-521.

[13] M. Santos, On non-existence of continuous Markov equilibria in competitive-market economies, J. Econ. Theory (2001), this issue.

[14] N. Stokey and S. Rebelo, Growth effects of flat-rate taxes, J. Polit. Economy 103 (1995), 519-550. 